\section{Primary renal synovial sarcoma}

\author{
Girish D. Bakhshi, ${ }^{1}$ Arshad S. Khan, ${ }^{1}$ \\ Aftab S Shaikh, ${ }^{1}$ \\ Mohammad Ashraf A. Khan, ${ }^{1}$ \\ Mohammad Adil A. Khan, ${ }^{1}$ \\ Nilofar M. Jamadar ${ }^{2}$
}

${ }^{1}$ Department of Surgery and ${ }^{2}$ Department of Radiology, Grant Medical College \& Sir J.J. Group of Hospitals, Mumbai, India

\begin{abstract}
Primary Renal Sarcoma is rare tumor comprising only $1 \%$ of all renal tumours. Synovial sarcomas are generally deep-seated tumors arising in the proximity of large joints of adolescents and young adults and account for $5-10 \%$ of all soft tissue tumours. Primary synovial sarcoma of kidney is rare and has poor prognosis. It can only be diagnosed by immunohistochemistry. It should be considered as a differential in sarcomatoid and spindle cell tumours. We present a case of 33-year-old female, who underwent left sided radical nephrectomy for renal tumour. Histopathology and genetic analysis diagnosed it to be primary renal synovial sarcoma. Patient underwent radiation therapy and 2 years follow up is uneventful. A brief case report with review of literature is presented.
\end{abstract}

\section{Introduction}

Primary renal synovial sarcoma (PRSS) was initially described by Faria et al. in 2000 which was previously included in embryonal sarcomas of the kidney. ${ }^{1-3}$ Detection of characteristic cytogenetic abnormality $t(X, 18)$ within this group of tumors led to the recognition of a renal synovial sarcoma. These tumors harbour characteristic translocation $\mathrm{t}(\mathrm{X} ; 18)$ (p11; q11) described for synovial sarcoma resulting in fusion proteins like SYT-SSX1, SYT-SSX2 and SYTSSX4. ${ }^{1,2,4}$ Despite all the features, the nature of the tumour and natural history, the management of this tumour is still a matter of further investigation. There are only 51 cases of primary renal synovial sarcomas reported till date. ${ }^{4,5}$ We present a case of PRSS which was diagnosed by genetic analysis.

\section{Case Report}

A 33-year-old female presented with a mass in left upper abdomen. She had an episode of hematuria and mild pain in left upper abdomen for 2 months. The size of the mass had increased in 2 months. Clinical examination revealed $20 \times 10 \mathrm{~cm}$ mass in left lumbar region, extending into left iliac region. Computed tomography (CT) scan showed a mass of approximately $20 \times 12 \times 11.5 \mathrm{~cm}$ arising from the lower pole of the left kidney. It was extending from left renal fossa to left iliac region with areas of hemorrhages and necrosis in it (Figures 1 and 2). A provisional diagnosis of renal cell carcinoma was made. Metastatic work up confirmed it to be a localised tumor. Patient underwent left radical nephrectomy (Figure 3). On histopathological examination; monophasic primary renal synovial sarcoma was diagnosed with capsular infiltration and involvement of Gerota's fascia (Figure 4). Genetic analysis confirmed presence of SYT-SSX2 $(X, 18)$ translocation. Her postoperative period was unremarkable. She received external radiotherapy with Cobalt gamma rays of $4500 \mathrm{cGy}$ in 25 cycles. Her 2 years follow up has shown her to be disease free.

\section{Discussion}

Primary kidney sarcomas are rare neoplasms that account for $1 \%$ of all malignant renal tumors. ${ }^{1}$ Leiomyosarcoma is the most frequent type of renal sarcoma, comprising about $40-60 \%$ of all cases. ${ }^{1}$ Other sarcomas that involve the kidney include rhabdomyosarcoma, malignant fibrous histiocytoma, fibrosarcoma, angiosarcoma, hemangiopericytoma, and rarely synovial sarcoma. Synovial sarcomas are commonly seen in the proximity of large joints. They can be observed in unexpected sites, such as thoracic and abdominal wall, head and neck region, including pharynx and larynx, retroperitoneum, bone, as well as visceral organs, such as lung, pleura, or prostate. This shows that synovial sarcomas may develop de novo at other sites where synovial membrane is not present. Hence histogenesis is uncertain as in PRSS. The occurrence of a primary renal sarcoma is rare and is considered in the differential diagnosis of sarcomatoid renal tumors after judiciously ruling out sarcomatoid renal cell carcinoma and primary retroperitoneal soft tissue sarcomas with secondary renal involvement, both of which can resemble a primary renal sarcoma. ${ }^{1}$ Immunohistocytochemistry and genetic analysis helps in establishing diagnosis of most of the tumors and in case of dubious histopathology and immunocytochemistry, genetic analysis should be considered. ${ }^{1,3}$

PRSS generally presents in adults between the ages of 15 and 71 years with equal incidence in both the sexes. The clinical symptoms mostly include abdominal pain and hematuria as was seen in our patient. Despite the limited followup data and the small number of cases reported,
Correspondence: Girish D. Bakhshi, Devneeti, Plot-61, Sector-7 Koper khairane, Navi Mumbai400709, Maharashtra, India.

Tel: +91.9820218198 .

E-mail: gdbakhshi@yahoo.com

Key words: renal tumor, synovial sarcoma.

Received for publication: 22 December 2011.

Revision received: 14 February 2012.

Accepted for publication: 2 March 2012.

Acknowledgement: Our sincere thanks to Padmashree Dr. T.P. Lahane, Dean, and Professor M.B.Tayade Head, Department of Surgery, Grant Medical College \& Sir J.J. Group of Hospitals for guiding and permitting us to publish this article.

Contributions: GDB, concept and design, manuscript final approval; ASK, ASS, draft preparation and revision; MAK, MAK, NMJ, data acquisition and analysis.

Conflict of interest: the authors declare no potential conflicts of interest.

This work is licensed under a Creative Commons Attribution NonCommercial 3.0 License (CC BYNC 3.0).

(C) Copyright G.D. Bakhshi et al., 2012

Licensee PAGEPress, Italy

Clinics and Practice 2012; 2:e44

doi:10.4081/cp.2012.e44

this tumor has already proved to have a generally aggressive behavior. ${ }^{1,3}$ Renal synovial sarcomas present as large masses ranging from 1 to $35 \mathrm{~cm}$, usually between 5 and $20 \mathrm{~cm}$ in diameter. They are frequently associated with cysts with smooth walls. Cysts are lined by mitotically inactive polygonal eosinophilic cells with apically oriented nuclei (hobnailed epithelium). Microscopically, tumors are characterized by mitotically active, monomorphic plump spindle cells with indistinct cell borders growing in short, intersecting fascicles. ${ }^{3-5}$ Synovial sarcomas are histomorphologically grouped into 3 types; monophasic synovial sarcoma (MSS), biphasic synovial sarcoma (BSS) and poorly differentiated synovial sarcoma (PDSS) which consists of $20 \%$ and has poorer prognosis. The monophasic spindle cell variant consists of spindle cells with little or no evidence of epithelial differentiation. MSS appears frequently than the biphasic form, with both types sharing the same clinical, ultrastructural, and molecular features. Unlike the BSS patterns, the recognition of monophasic fibrous synovial sarcoma and PDSS subtypes is often a diagnostic challenge for pathologists because they may easily be confused with other spindle to round cell sarcomas, especially malignant peripheral nerve sheath tumors, fibrosarcoma, leiomyosarcoma, liposarcoma, and Ewing sarcoma. ${ }^{1,4-7}$ 
Synovial sarcomas are characterized by the translocation $\mathrm{t}(\mathrm{x} ; 18)$ (p11.2; q11.2). The breakpoint of this translocation fuses the SYT gene from chromosome 18 to one of three homologous genes, SSX1, SSX2, and SSX4 on the X chromosome. Coexpression of two forms of SYT messenger RNA, I-SYT and N-SYT, has been described in both normal tissues and synovial sarcomas. Although both fuse with SSX, N-SYT fusion products predominate in normal tissues, while the ISYT isoform is consistently overexpressed in synovial sarcomas. The SYT-SSX gene is thought to function as an aberrant transcriptional regulator. The nature of the chimeric gene appears to be of prognostic importance, as metastasis-free survival is significantly higher in patients with SYT-SSX2 compared to SYT-SSX1 fusion genes. SYT-SSX1 is associated with biphasic tumors, while SYT-SSX2 is associated with monophasic tumors that lack glandular epithelial differentiation. However, not all reports support a worse outcome for patients with biphasic as compared to monophasic histology. In addition to these cytogenetic changes, Bcl-2 expression, as assessed by immunohistochemistry, has been

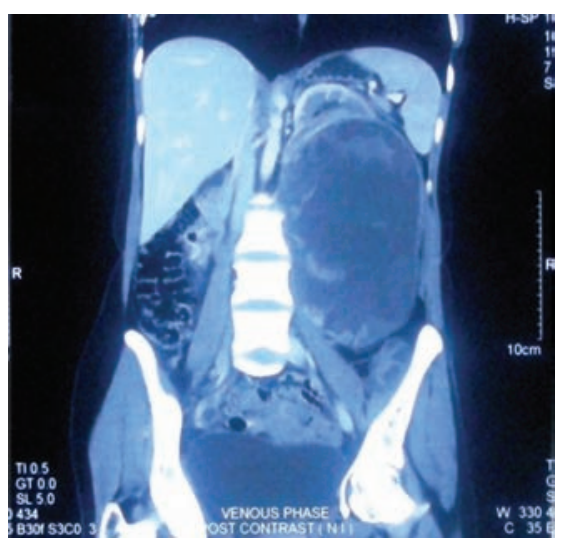

Figure 1. Computed tomography of abdomen showing mass arising from lower pole of kidney measuring $20 \times 12 \times 11.5 \mathrm{~cm}$ which shows areas of haemorrhage and necrosis.

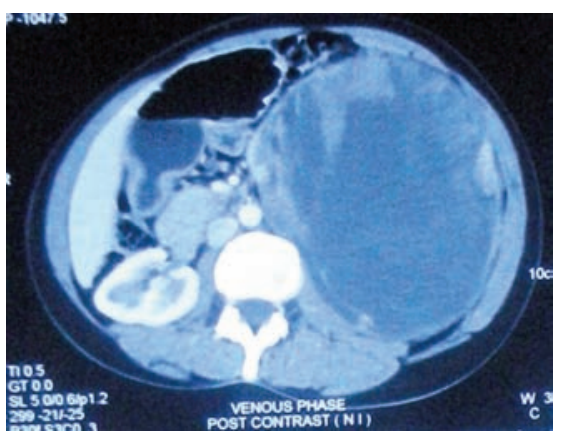

Figure 2. Transverse section of computed tomography image of Figure 1. reported to be an almost general constitutive alteration of synovial sarcomas 4,6,8. We also confirmed expression of $\mathrm{Bcl}-2$ protein which was negative, by using both genetic and Western blot analysis. The $\mathrm{Bcl}-2$ gene expression in a patient with SYT-SSX2 may indicate ineffectiveness of chemoradiation after surgery. ${ }^{2,7,9}$ Ultrasound and computed tomography may be one of the means of diagnosis, but may not diferentiate between, renal synovial sarcoma and other sarcomas. It shows only areas of necrosis and haemorrhages which is suggestive of malignancy.,3 The treatment protocol of PRSS consists of adjuvant ifosfamide based chemotherapy programs in conjunction with radical nephrectomy. High-dose ifosfamide-based chemotherapy has been used as neoadjuvant and adjuvant therapy in patients with localized or advanced soft tissue synovial sarcomas, and favourable results have been reported. ${ }^{2,3}$ Radiotherapy is also effective in patients with Bcl-2 negative expression as it was in our patient. ${ }^{2,7} \mathrm{~A}$ study reported tumor-free survival in $90 \%$ patients after a 37 -month-follow-up with localized soft tissue synovial sarcoma. ${ }^{7-9}$ Other study reported complete remission using a

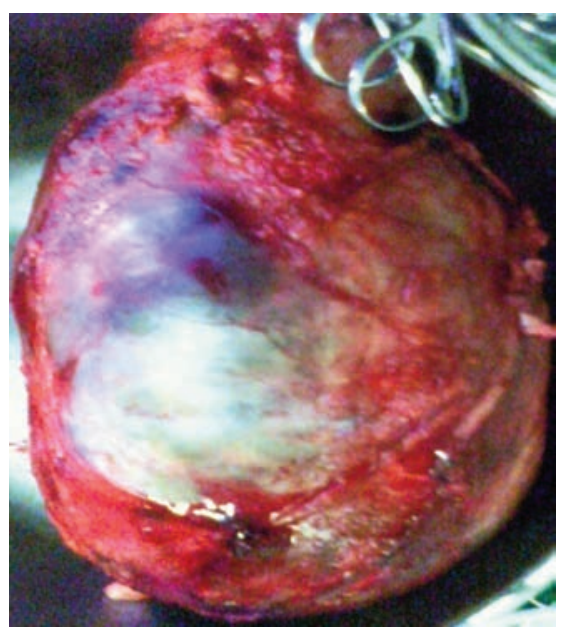

Figure 3. Gross specimen of left nephrectomy.

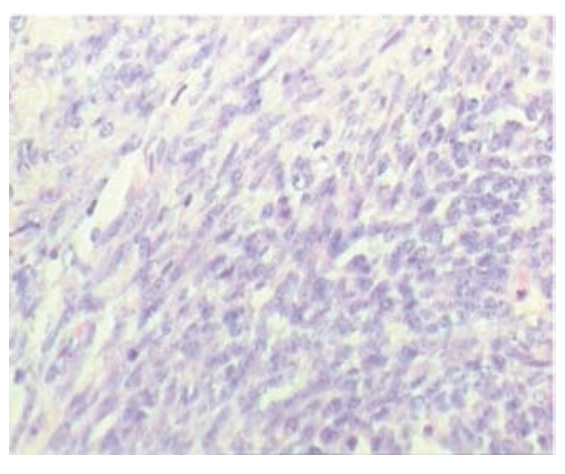

Figure 4. Microscopic picture of tumour showing spindle cells with capsular involvement (Hematoxylin \& Eosin stain 20X). doxorubicin and ifosfamide protocol in a PRSS patient developing metastases in the lung in the fourth month following radical nephrectomy. ${ }^{2}$ However, currently, there is no definite consensus regarding the use of chemotherapy for patients with PRSS. More studies are required to develop a definite plan of treatment.

\section{Conclusions}

PRSS is a rare tumour of kidneys which can be diagnosed on histopatholgy combined with immunohistochemistry and genetic analysis. Surgery is the mainstay of treatment. Radiotherapy can be useful as an adjuvant therapy in presence of local spread. Chemotherapy may be beneficial in distant metastases. However, more studies are required for optimising treatment of this rare tumour for better prognosis.

\section{References}

1. Zia Z, Al Shakarchi J, Bagree R, et al. Case report: primary synovial sarcoma of kidney. The Internet Journal of Urology 2007;2:152832.

2. Park SJ, Kim HK, Kim CK, et al. A case of renal synovial sarcoma: complete remission was induced by chemotherapy with doxorubicin and ifosfamide. Korean J Intern Med 2004;19:62-5.

3. Argani P, Faria PA, Epstein JI, et al. Primary renal synovial sarcoma: molecular and morphologic delineation of an entity previously included among embryonal sarcomas of the kidney. Am J Surg Pathol 2000;24:1087-96.

4. Tsai YK, Lin CJ, Wang JH, et al. Primary synovial sarcoma of the kidney: a case report. Chin J Radiol 2004;29:359-63.

5. Murphy WM, Beckwith JB, Farrow GM. Tumors of the kidney, bladder, and related urinary structures. In: Atlas of tumor pathology, 3rd series, Fascicle 11. Washington, DC: AFIP; 1994. pp.219-30.

6. Mancuso, T, Mezzelani, A, Riva, C, et al. Analysis of SYT-SSX fusion transcripts and bcl-2 expression and phosphorylation status in synovial sarcoma. Lab Invest 2000;80:80513.

7. Kawai, A, Woodruff, J, Healey, JH, et al. SVTSSX gene fusion as a determinant of morphology and prognosis in synovial sarcoma. N Engl J Med 1998;338:153-60.

8. Skytting B, Nilsson G, Brodin B, et al. A novel fusion gene, SYT-SSX4, in synovial sarcoma. J Natl Cancer Inst 1999;91:974-5.

9. Paulino AC. Synovial sarcoma prognostic factors and patterns of failure. Am J Clin Oncol 2004;27:122-7. 